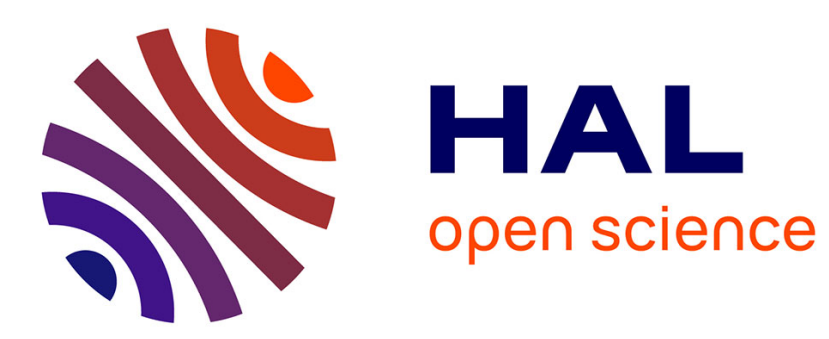

\title{
CORRELATEUR A MEMOIRE A ONDES DE SURFACE.
}

\author{
C. Maerfeld, Hélène Gautier
}

\section{To cite this version:}

C. Maerfeld, Hélène Gautier. CORRELATEUR A MEMOIRE A ONDES DE SURFACE.. Journal de Physique Colloques, 1979, 40 (C8), pp.C8-243-C8-248. 10.1051/jphyscol:1979842 . jpa-00219547

\section{HAL Id: jpa-00219547 https://hal.science/jpa-00219547}

Submitted on 1 Jan 1979

HAL is a multi-disciplinary open access archive for the deposit and dissemination of scientific research documents, whether they are published or not. The documents may come from teaching and research institutions in France or abroad, or from public or private research centers.
L'archive ouverte pluridisciplinaire HAL, est destinée au dépôt et à la diffusion de documents scientifiques de niveau recherche, publiés ou non, émanant des établissements d'enseignement et de recherche français ou étrangers, des laboratoires publics ou privés. 
CORRELATEUR A MEMOIRE A ONDES DE SURFACE,

C. Maerfeld et H. Gautier.

THOMSON-CSE, Division Activités sous-marines, Chemin des Travails, 06802 Cagnes-sur-mer.

Résumé. - Les interactions acoustiques non-linéaires en ondes de surface ont été mises à profit pour réaliser des convoluteurs et des corrélateurs à mémoire. Le but de ce papier est de faire un bref survol des principes de fonctionnement et des performances de ces dispositifs, ainsi que de l'état d'avancement des recherches en cours.

Summary. - The acoustical non linear surface wave interactions are used to provide memory convolutors and correlators. The purpose of this paper is to give a brief sketch of the basic principles of working, the accuracy of these devices and the state of the works in progress.

\section{Corrélateur à mémoire à ondes de surface.-}

En 1969, L.0. Svaasand /1/ et C.F. Quate, R.B. Thomson /2/ ont montrë comment on pouvait mettre à profit les non-linéarités intrinsèques des matériaux pour effectuer le produit de convolution de deux signaux $F(t) \cos \omega_{1} t$ et $G(t) \cos \omega_{2} t$. Le schéma de principe apparaît sur la Figure 1. Les deux signaux sont appliqués à deux transducteurs en forme de peignes interdigitês gravês sur un substrat piézoélectrique (Quartz ou Niobate de Lithium). Ils sont alors transformés en deux ondes contradirectives $F\left(t-\frac{z}{v}\right)$. $\cos \left(\omega_{1} t-k_{1} z\right)$ et $G\left(t+\frac{z}{v}\right) \cdot \cos \left(\omega_{2} t+k_{2} z\right)$.
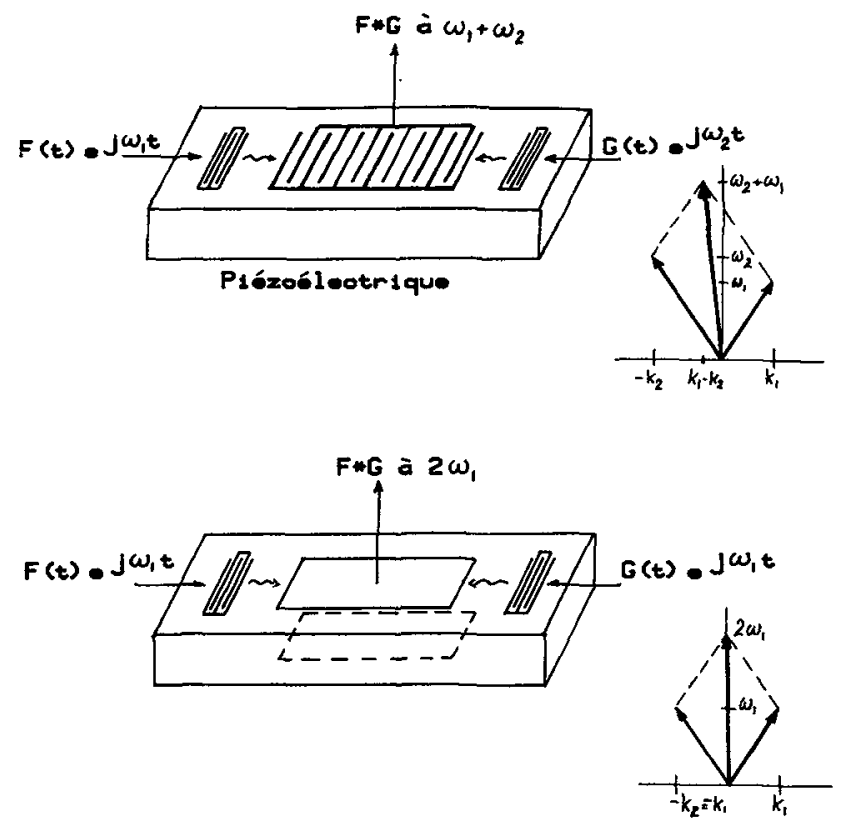

FIGURE 1 : Schéma de principe des convoluteurs.
L'interaction non-linéaire fait apparaitre des termes produits de fréquences temporelles $\omega_{1} \pm \omega_{2}$ et de nombres $d^{2}$ ondes $k_{1} \mp k_{2}$. Pour recueillir le signal résultant, un peigne de longueur $L$ est gravé dans la zone d'interaction. Sa période $p$ est telle que $2 \pi=k \mp k$. Alors la sortie est de la forme :

$S(t)=\exp j\left(\omega_{1}+\omega_{2}\right) t \int_{L} F\left(t-\frac{z}{V}\right) G\left(t+\frac{z}{V}\right) d z$

On reconnait le produit de convolution des 2 signaux $F$ et $G$ mais comprimé cans le temps d'un facteur 2 .

Les convoluteurs les plus généralement utilisés sont du type"dēgénêrés", c'est-à-dire tels que $\omega_{1}=\omega_{2}=\omega$. Alors, le transducteur de réception est une simple plaque métallique uniforme (Fig. 1b) qui recueille un sianal ã la fréquence $2 \omega$.

De nombreuses équipes se sỏnt alors intéressées à ce type de dispositif. On a tout d'abord cherché à rendre les effets non-1inéaires plus importants par exemple en utilisant l'interaction de 1 'onde acoustique avec un semiconducteur d'après l'idée originale de W.C. Wang $/ 3 /$.

Plusieurs dispositîfs ont été envisagés comme on peut le voir sur la Fig. 2 : le semiconducteur est placé ã proximité du substrat piézoêlectrique (Fig. 2a). Le substrat est à la fois semiconducteur et piézoélectrique (exemple CdS ou AsGa) (Fig. 2b). Le Silicium est recouvert d'une couche piézoélectrique permettant la génération des ondes de surface et le couplage avec le semiconducteur (Fig. 2c) /4/. Enfin, on peut utiliser le couplage 
avec des éléments discrets d'un circuit extérieur /5/ (Fig. 2d).
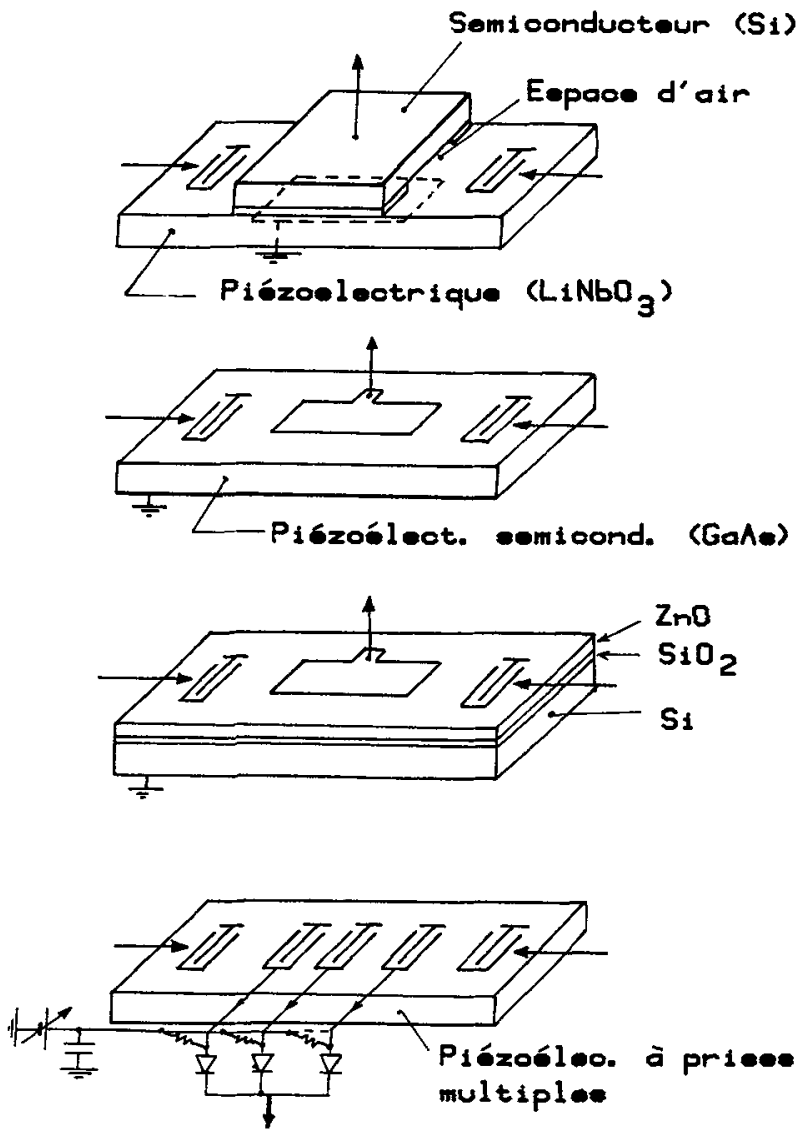

FIGURE 2 : Différents types de convoluteurs à ondes de surface et semiconducteur

2a) Couplage avec espace d'air

2b) Couplage dans un semiconducteur piēzoélectrique

2c) A couche piézoélectrique déposée

2d) A prise et diodes extérieures.

Les convoluteurs sont caractērisés par leur

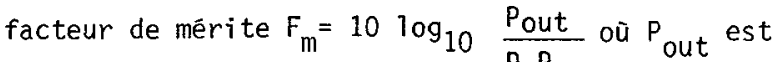
1a puissance recueillie en sortief ${ }^{P} G$ pour des puissances électriques $P_{F}$ et $P_{G}$ à $T$ 'entrée. Toutes ces puissances sont exprimées en $\mathrm{mW}, F_{m}$ est en $\mathrm{dBm}$. Il y a également lieu de considérer le nombre de points des signaux que 1 'on peut traiter, c'est-àdire le produit de la bande passante $B$ par leur longueur $T$. Des facteurs de mérite importants ne sont en général pas compatibles avec des BT élevés, car alors des phénomènes de saturation apparaissent, des distorsions de phase 1 imitent la bande passante, des transferts d'énergie sur des modes d'ordre élevé augmentent 1 'atténuation et limitent $1 a$ durée $T$. Pour des B.T $\geqslant 500, F_{m}$ est de 1 'ordre de $-60 \mathrm{dBm}$ comme le montre le Tableau 1. Dans ces conditions, le couplage avec le semiconducteur n'apparait pas comme absolument nécessaire : un convoluteur basē sur les effets non-linéaires intrinsèques, mais comportant un compresseur de faisceau qui concentre $T$ 'énergie incidente sur une voie étroite de manière à augmenter la densitê d'énergie et donc le rendement, donne des performances semblables en $F_{m}$, mais présente une uniformité d'interaction presque parfaite (Fig. 3 et tableau I). En outre, aucune saturation de la bilinéarité n'a pu y être observêe jusqu'à des puissances d'entrée de l'ordre du W.

Le tableau I montre d'ailleurs les performances comparées des meilleurs convoluteurs connus. La puissance de sortie maximale détermine la dynamique de sortie; en effet la puissance minimale est limitée par le bruit du prëamplificateur de sortie, doit $F k \in B, F$ facteur de bruit, $K$ constante de Boltzman, $\Theta$ température absolue.

2. Corrélateur à mémoire.- Les effets non-linéaires acoustiques ont également été utilisés pour rêaliser une mise en mémoire d'information. Les premières expëriences à ce sujet ont êté baptisées "échos de phonons" $/ 6,7,8,9 /$. Les mêmes mécanismes que précédemment agissant sur des ondes de volume engendrent dans un piézoélectrique semiconducteur une modulation spatiale du taux de remplissage des pièges à électrons responsable d'un effet de mémoire. Mais c'est vraiment en 1974 que 1'idée d'utiliser un réseau de diodes est apparue $/ 10 /$, et qu'il a êté montré que cette fonction mémoire ajoutée à un convoluteur, permet d'obtenir un corrêlateur fonctionnant en mode asynchrone, les diodes étant de type Schottky ou pn /11, 12/. C'est le corrélateur à mémoire dont nous allons décrire le fonctionnement et les performances.

Le schëma est celui de la Fig. 4. Un réseau de diodes, dont le pas $p=12,5 \mu$ est notablement plus petit que la demi-longueur d'onde, et placé à proximité d'un substrat de Niobate de Lithium. L'espace d'air qui sépare les deux milieux, et qui est d'environ $2000 \AA$, est assuré à 1 'aide de petits plots de quelques microns de diamètre gravés sur le substrat et répartis aléatoirement sur la longueur d'interaction.

Le fonctionnement du dispositif est le suivant. Le signal $G(t)$ coswt, de bande passante 


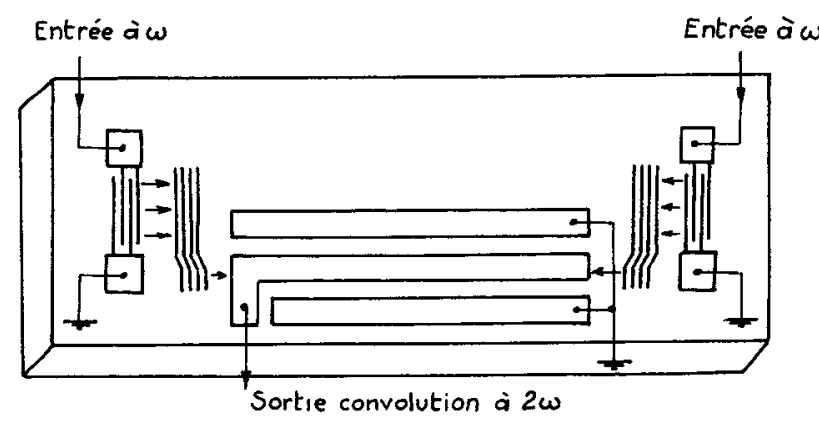

FIGURE 3 : Shéma d'un convoluteur à concentrateur de faisceau. polarise en direct et bloquant les autres alternances. Il en résulte un déplacement continu de charges qui les polarise en inverse à la valeur crête du potentiel d'excitation.

Le potentiel d'excitation a deux origines : le potentiel associē à l'onde de surface du fait de de la nature piézoélectrique du substrat, que l'on écrit $G\left(t-\frac{z}{v}\right) \cos (\omega t-k z)$, et le potentiel appliqué à 7 a plaque.

Dans le cas de l'écriture flash et si le temps de charge des diodes est suffisamment rapide, les

\begin{tabular}{|c|c|c|c|c|}
\hline TYPE DE CONVOLUTEUR & $\begin{array}{l}\text { LiNb03 } \\
\text { (+Comproseours) }\end{array}$ & $S_{1 / A 1 R / L i N b O G}$ & \multicolumn{2}{|c|}{$\mathrm{ZnO} / \mathrm{S}_{102 / \mathrm{S}_{1}}$} \\
\hline FREQUENCE CENTRALE MHz & 180 & 300 & 125 & 225 \\
\hline BANDE PASSANTE MHz & 40 & 100 & 22 & 30 \\
\hline DUREE TRAITEMENT $\mu$ & 12 & 20 & 3 & 2.3 \\
\hline PRODUIT BT & 480 & 2000 & 86 & 69 \\
\hline UNIFORMITE dB & \pm .3 & \pm 1 & \pm .5 & - \\
\hline FACTEUR MERITE $\mathrm{dBm}$ & -70 & -65 & -70 & -57 \\
\hline PUISS. MAX. ENTREE dBm & $>30$ & 30 & 28 & 24 \\
\hline SORTIE dBm & $>-10$ & -5 & -25 & -10 \\
\hline REFERENCES & GAUT IER-MAERFELD & REIBLE ot al & $\begin{array}{l}\text { KIND } \\
\text { ot al }\end{array}$ & $\begin{array}{l}\text { ELIOTT } \\
\text {-t al }\end{array}$ \\
\hline
\end{tabular}

TABLEAU I : Performances des convoluteurs.

$B$ de durée $T$, est appliqué au transducteur $A$. Quand 1 'onde qui en résulte $G(t-z / v) \cos (\omega t-k z)$ est dans la zone d'interaction, on applique sur

la plaque $P$ :

- Soit une impulsion de Dirac $w(t)$ dont l'amplitude atteint $100 \mathrm{~V}$ et dont la durēe $\tau$ est infērieure à la demi-période porteuse soit $\tau<\pi / \omega$ : c'est l'écriture"flash".

- Soit une impulsion avec porteuse $w(t) \cos \omega t$, de même fréquence que le signal à inscrire et dont la durée $\tau$ est inférieure à $1 / B$, c'est 1 'écriture paramétrique.

Le mécanisme d'écriture se comprend aisément si l'on remarque que les diodes agissent en détecteur crête : elles sont passantes pour les alternances pour lesquelles le potentiel appliqué les charges accumulées par les diodes engendrent donc une polarisation inverse de la forme :

$V(x)=W+G(-z / v) \cos k z$

Dans le cas de l'écriture paramétrique, la polarisation finale des diodes est de la forme :

$$
\begin{aligned}
V(x) & =\max (|W \cos (\omega t)+G \cos (\omega t-k z)|) \\
& =W^{2}+G^{2}+2 G W \cos k t
\end{aligned}
$$

qui se rêduit à, si $W \gg G$ :

$$
V(x)=W+G(-z / v) \cos k z
$$



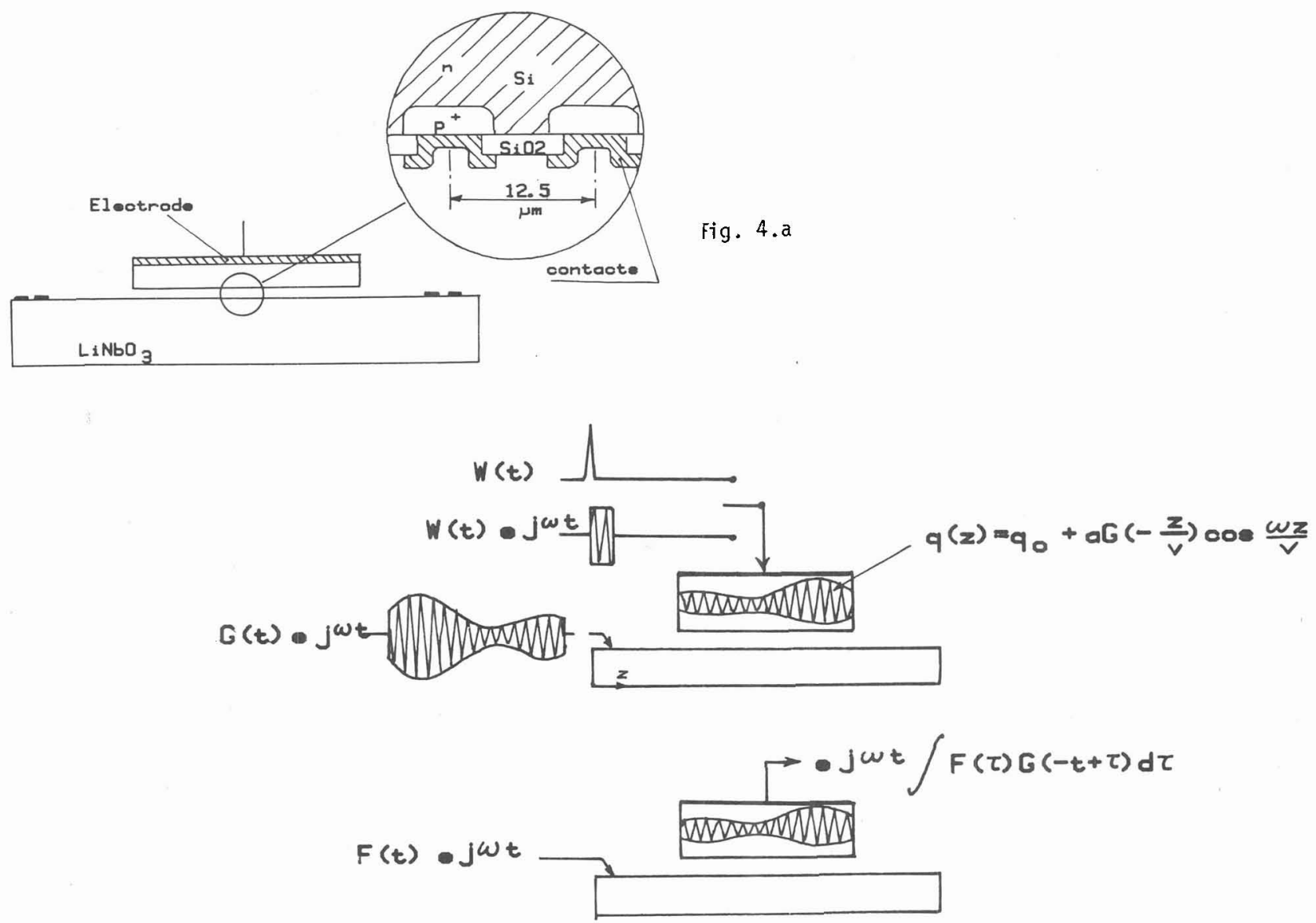

FIGURE 4: Schêma de corrểateur à mêmoire

Fig. 4.b

4a) Schëma

4b) Principe

On obtient donc le même réseau de charges stockēes dans les deux cas. Ce rêseau disparaît lentement par suite du courant inverse (faible) des diodes. C'est ainsi que des durées de mémorisation de plusieurs secondes ont pu être observées avec des pn $/ 12 /$.

Pendant la durée de mémorisation, le potentiel existant à l'interface semiconducteur-piézoêlectrique est identique et opposé à celui de 7 'onde incidente : I'onde est comme "figée ".

Le même mécanisme que celui du convoluteur peut alors être mis à profit : on fait un battement non-linéaire entre l'onde figée et une autre onde dite de Tecture $F(t)$ cos wt de même fréquence w et appliquée au même peigne. Le signal de sortie recueilli sur la plaque à la même fréquence $\omega$ est :

$S(t)=\cos \omega t \int_{L} F(t-z / v) G(-z / v) d z$
C'est la corrélation entre $F$ et $G$, sans compression de temps. La figure 5 donne un exemple de démonstration : le signal stockē est un code de Barker de 13 bits à $120 \mathrm{MHz}$ de fréquence centrale. Le même signal est appliqué $10 \mathrm{~ms}$ plus tard et 1 'on obtient en sortie l'autocorrélation du code de Barker qui est représentêe sur la Fig. 5 .

Un tel dispositif ainsi décrit représente un filtre entièrement programmable dont il suffit d'inscrire la réponse impulsionnelle. Ce dispositif est particulièrement adpatê au traitement des signaux radars dans la mesure où la durēe $T$ des signaux stockês est limitēe à une dizaine de microsecondes et oū par contre, Ta bande passante B pourrait atteindre $100 \mathrm{MHz}$. C'est pourquoi il est étudiē par de nombreuses équipes qui cherchent à augmenter $T$, B et 1 'efficacité de 1 'interaction. Les études ont pour 1 'instant surtout portẽ sur la nature et la géométrie des diodes. On peut mesurer les résultats de la façon suivante. 

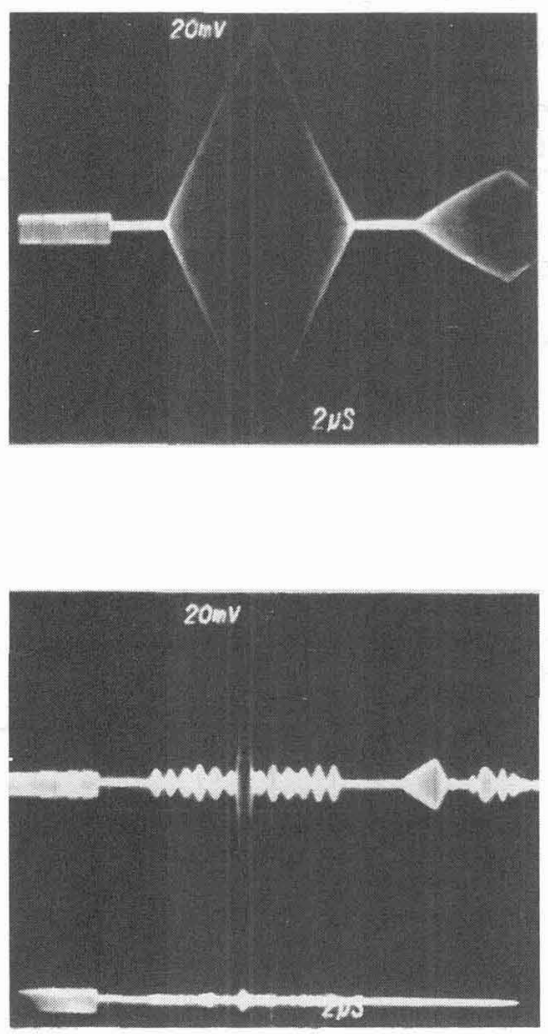

FIGURE 5 : Maquette de corrélateur à diode Schottky

Autocorrêlation de signaux de $3,5 \mu \mathrm{s}$ exécutée $1 \mathrm{~ms}$ après l'êcriture

a) Signaux $\mathrm{CW}$

b) Code de Braker à 13 bits.

\section{a) Schotttky ou on pn?}

Les diodes Schottky ont des durées de commutation de 1 'ordre de la nanoseconde. Il est donc possible avec de telles diodes d'inscrire des signaux de très grande bande passante ( $>100 \mathrm{MHz}$ ) Par contre, les courants inverses qui sont d'origine thermo-ionique sont relativement élevês : les durëes de mémorisation sont 1 imitées à environ $100 \mathrm{~ms}$ sur substrat de Silicium /13, 14/.

Les diodes pn ont des durées d'écriture de plusieurs dizaines de nanosecondes. Seule l'êcriture de type paramétrique est utilisable, la bande passante maximale stockable étant limitēe par ce fait à environ 20 ou $30 \mathrm{MHz}$. Par contre, le faible courant inverse autorise des durēes de mémorisation dépassant $1 a$ seconde $/ 14 /$.

\section{b) Rësisstivivitể_du subst_trat semiconducteur.}

Ce rendement non-linéaire est d'autant plus êlevé que la résistivité du substrat est plus êTevée. Cependant, pour des rēsistivités supērieures à $100 \Omega-\mathrm{cm}$, les zones de déplētion de chacune des diodes risquent de se boucher rendant impossible le stockage d'un réseau : pratiquement, la résistivité optimale se situe aux alentours de $10 \Omega-\mathrm{cm}$.

\section{c) Géométrịe_des diodedes.}

L'onde stockée est en fait échantillonnée. Le nombre d'échantillons par longueur d'onde doit être supérieur à 2 si on ne veut pas apercevoir 7 'effet de cet échantillonnage. Ceci exige des pas de $4 \mu$ pour stocker des signaux de $300 \mathrm{MHz}$ de frëquence centrale. De tels pas, compte tenu du fait que les bandes relatives maximales réalisables efficacement en onde de surface ne dépassent pas $30 \%$, autorisent des stockages de signaux de $100 \mathrm{MHz}$ de bande (en diode Schottky). Dans le sens perpendiculaire à la propagation, le pas peut être plus grand. On utilise alors une diode de forme oblongue devant limiter les courants de fuite latéraux $/ 13 /$.

d) Silicium ou AsGa. On a rēcemment reảisé des diodes Schottky sur AsGa (contact Platine). La hauteur de barrière $V_{b i}$ du contact Platine-AsGa étant de $0,95 \mathrm{~V}$, on doit logiquement s'attendre à un courant inverse plus faible que sur substrat Silicium ( $V_{b i}=0,55 \mathrm{~V}$ pour Mo/Silicium). Effectivement, des durées de stockage de $400 \mathrm{~ms}$ ont été observées $/ 15 /$. Ces diodes ont des vitesses d'écriture comparables aux diodes Schottky sur Silicium, et sont donc particuliērement intēressantes.

Le tableau II résume les performances ainsi obtenues. Les pertes du filtre en corré\}ation sont définies comme le rapport de la puissance du signal de sortie à la puissance du signal de lecture pour une puissance de signal à inscrire de $1 \mathrm{~W}$. On note que des signaux dont le BT dépasse la centaine ont pu être corrétés et que 1'on pense obtenir des BT de 1000 dans un proche avenir. Cependant, de nombreux problèmes restent encore à résoudre : l'uniformité d'interaction est à améliorer, 1a dynamique est actuellement trop limitée par un niveau de parasites anormalement êlevê .... 


\begin{tabular}{|c|c|c|c|c|c|c|}
\hline \multirow[b]{2}{*}{ DIODE TYPE } & \multicolumn{4}{|c|}{ DEMONSTRATED } & \multicolumn{2}{|c|}{ LIMITS } \\
\hline & PN & & $\begin{array}{l}\text { CHOTTKY } \\
\text { ICIUM }\end{array}$ & GaAe & SCHOTTKY & PN \\
\hline DIODE PERIODICITY ( $\mu \mathrm{m})$ & 12.5 & 12.5 & 3. 8 & 12.5 & 3.5 & \\
\hline CENTRE FREQUENCY $\left(\mathrm{MHz}_{\mathbf{z}}\right)$ & 120 & 120 & 145 & 120 & 300 & \\
\hline BANDWIDTH $\quad\left(M H_{z}\right)$ & 20 & 20 & 30 & 20 & 100 & \\
\hline SIGNAL DURATION (HS) & 6 & 6 & 10 & 5 & $10-20$ & \\
\hline CORRELATION FILTER LOSS (dB) & -50 & -50 & -50 & -70 & - & \\
\hline SIGNAL / SPURIDUS (dB) & 25 & 25 & 30 & 25 & 40 & \\
\hline STORAGE TIME & $1-10 \mathrm{~s}$ & $80 \mathrm{mS}$ & $40 \mathrm{~ms}$ & $400 \mathrm{mS}$ & 0.45 & 15 \\
\hline REFERENCES & 1 & 1.2 & 3 & 4 & & \\
\hline
\end{tabular}

[1] Gaution-Maonfold

[2] Ingebrigteon

[3] Raleton ot al

[4] Gaution-Maonfold

TABLEAU II : Performances des corrêlateurs à mémoire.

Bibliographie

11 L.0. Svaasand "Interaction between elastic surface waves in piezoelectric materials" App 1. Phys. Lett. 15, 300-302 (1969).

12/ C.F. Quate and R.B. Thomson "Convolution. and correlation in real time with non linear acoustics "App7. Phys. Lett. 16, 494-496 (1970).

13/ W.C. Wang and P. Das "Surface wave convolvers via space charge non Tinearity "Proc. 1972 IEEE U1tras. Symp. 316-321 (1972).

14/ B.R. Khuri-Yakub and G.S. Kino "A monolithic Zinc-0xide on Silicon convolver "Appl. Phys. Lett. 25, 4, 188-190 (1974).

15/ T. M. Reeder, M. Gilden "Convolution and correlation by non linear interaction in a diode-coupled tapped delay line "Appl. Phys. Lett. 22, 8-10 (1973).

/6/ S.N. Popov, N.N. Krainik, Sov. Phys. Solid State 12, 2440 (1971).

17/ U.Kh. Kopvillem, B.P. Smolyakov, R.Z. Sharipov, JETP Lett. 13, 398 (1971).

18/ J. Joffrin, A. Levelut, "Boson echoes. A new tool to study phonon interaction "Phys. Rev. Lett. 29, 1325 (1972).

19/ N.S. Shirev, T.G. Kazyaka, Phys. Rev. Lett. 28,1304 (1972)
$110 /$ G. Kantorowicz, French patent $n^{\circ} 73.41234$ (1973).

111 K.A. Ingebritsen, R.A. Cohen, R.W. Mountain, Appl. Phys. Lett. 26, 596 (1975).

112/ C. Maerfeld, Ph. Defranould, P. Tournois, App]. Phys. Lett. 27, 577 (1975).

/13/ R.W. Ralston, J.H. Cafarella, S.A. Reible, E. Stern, Proceedings of IEEE Symposium On Sonics and U1traso.iics, p. 478 (1977).

/14/ H. Gautier, C. Maerfeld, P. Tournois, Proceedings of IEEE Symposium On Sonics and U7trasonics, p. 485 (1977).

115/ H. Gautier, C. Maerfe1d, P. Tournois Appl. Phys. Lett. 33, p. 517 (1978). 